Supporting Information for

\title{
Hydrogenation of aldehydes and ketones to corresponding alcohols with methylamine borane in neat water
}

Yifan Duan, Ruijiao Bai, Jun Tian, Ligong Chen and Xilong Yan*

School of Chemical Engineering and Technology, Tianjin University, Tianjin 30072, PR China.

E-mail: yan@tju.edu.cn

S1. ${ }^{1} \mathrm{H},{ }^{13} \mathrm{C},{ }^{11} \mathrm{~B}$ spectra of methylamine borane (MeAB)

${ }^{1} \mathrm{H}$ spectra of $\mathrm{MeAB}$

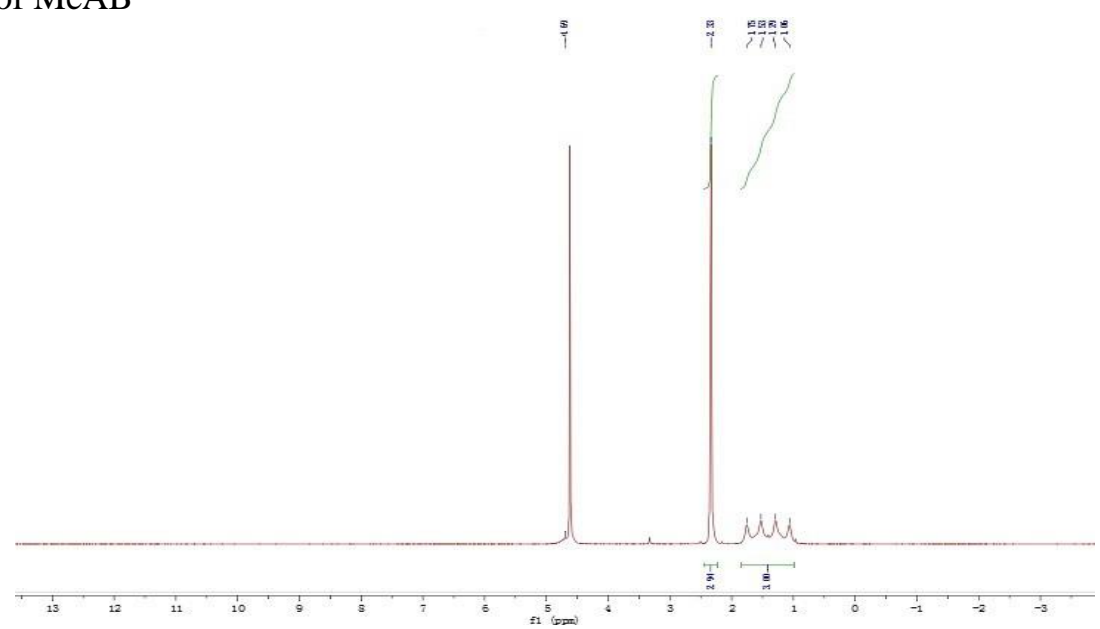

${ }^{13} \mathrm{C}$ spectra of $\mathrm{MeAB}$

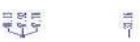

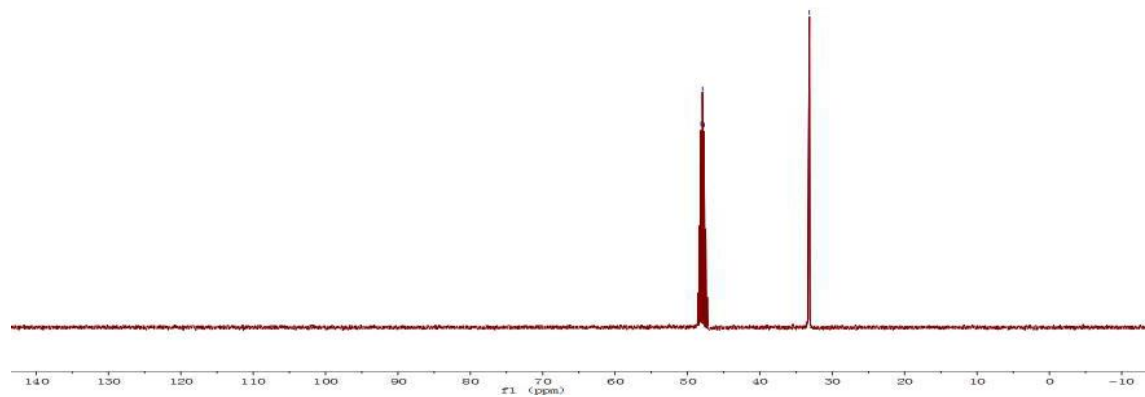

${ }^{11} \mathrm{~B}$ spectra of $\mathrm{Me} \mathrm{AB}$

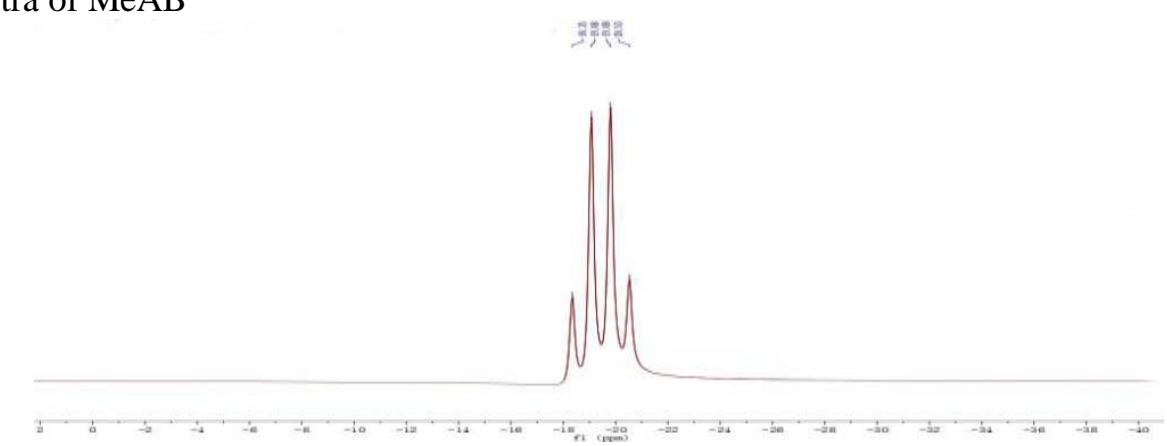


S2. XRD pattern of MeAB

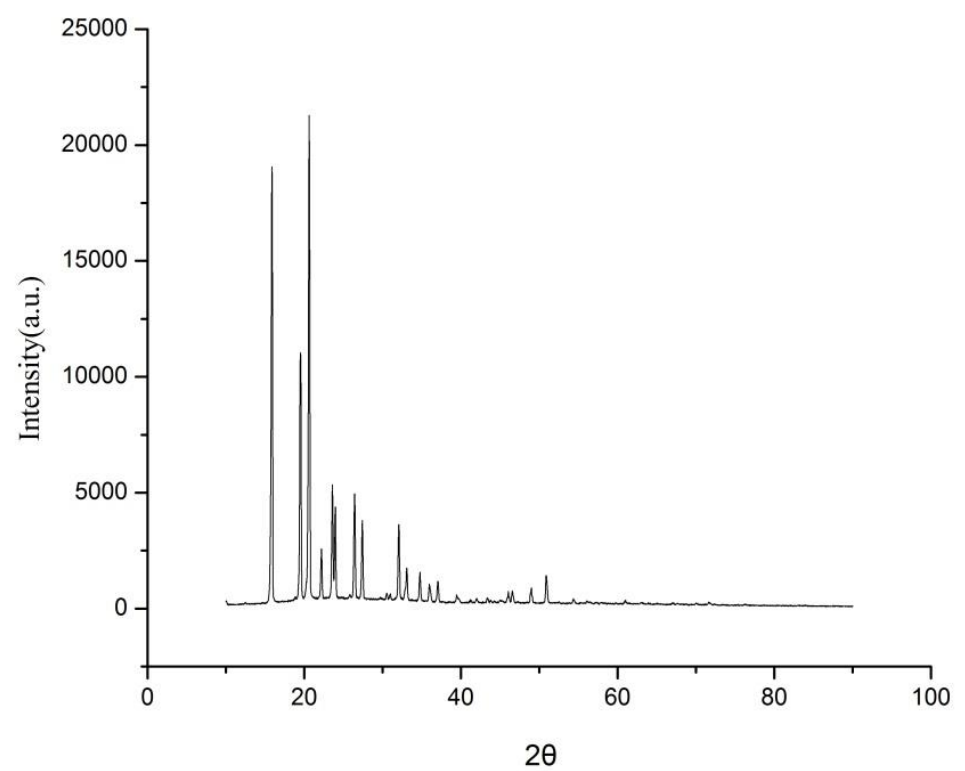

S3. Infrared spectra of MeAB

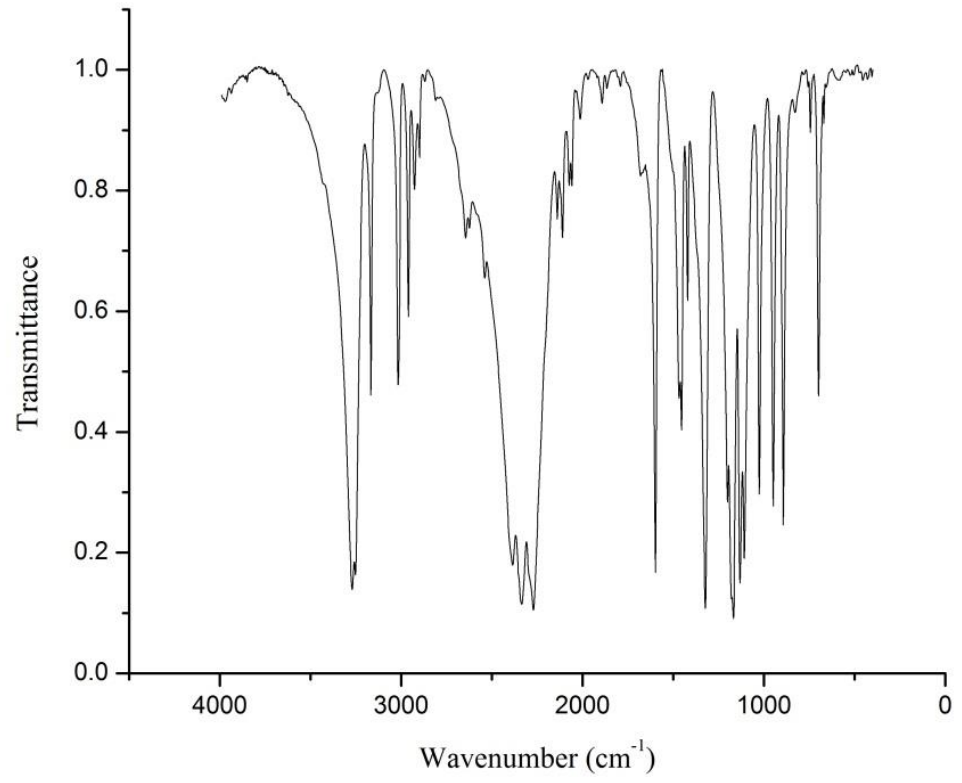

S4. Scheme 1

$3 \overbrace{\mathrm{R}^{\prime}}^{\mathrm{O}}+\mathrm{CH}_{3} \mathrm{NH}_{2} \mathrm{BH}_{3} \stackrel{\mathrm{H}_{2} \mathrm{O}}{\mathrm{rt}}{ }_{\mathrm{R}}^{\stackrel{\mathrm{OH}}{\mathrm{H}_{\mathrm{R}^{\prime}}}}+\mathrm{B}(\mathrm{OH})_{3}+\mathrm{CH}_{3} \mathrm{NH}_{2}$

$\mathrm{R}=$ aryl, alkyl; $\mathrm{R}^{\prime}=$ aryl, alkyl, $\mathrm{H}$ 
S5. ${ }^{1} \mathrm{H}$ and ${ }^{13} \mathrm{C}$ NMR characterization of products:

Phenylmethanol (entry 1, Table 1). ${ }^{1} \mathrm{H} \mathrm{NMR}\left(400 \mathrm{MHz}, \mathrm{CD}_{3} \mathrm{OD}, 25^{\circ} \mathrm{C}\right.$; TMS): $\delta$ $=4.61\left(\mathrm{~s}, 2 \mathrm{H} ; \mathrm{CH}_{2}\right), 7.26-7.35 \mathrm{ppm}(\mathrm{m}, 5 \mathrm{H} ; \mathrm{ArH}) ;{ }^{13} \mathrm{C} \mathrm{NMR}\left(100 \mathrm{MHz}, \mathrm{CD}_{3} \mathrm{OD}\right.$, $\left.25^{\circ} \mathrm{C} ; \mathrm{CD}_{3} \mathrm{OD}\right): \delta=63.89,126.67,126.95,128.02,141.24 \mathrm{ppm}$.

Cinnamyl alcohol (entry 2, Table 1). ${ }^{1} \mathrm{H}$ NMR (600 MHz, $\mathrm{CD}_{3} \mathrm{OD}, 25^{\circ} \mathrm{C}$; TMS): $\delta$ $=4.24\left(\mathrm{dd}, \mathrm{J}_{\mathrm{HH}}=6.0,1.8 \mathrm{~Hz}, 2 \mathrm{H} ; \mathrm{CH}_{2}\right), 6.38\left(\mathrm{dt}, \mathrm{J}_{\mathrm{HH}}=16.2,6.0 \mathrm{~Hz}, 1 \mathrm{H} ; \mathrm{CH}=\mathrm{CH}\right)$, $6.62\left(\mathrm{~d}, \mathrm{~J}_{\mathrm{HH}}=15.6 \mathrm{~Hz}, 1 \mathrm{H} ; \mathrm{CH}=\mathrm{CH}\right), 7.21-7.23\left(\mathrm{t}, \mathrm{J}_{\mathrm{HH}}=7.8 \mathrm{~Hz}, 1 \mathrm{H} ; \mathrm{ArH}\right), 7.30-$ $7.32\left(\mathrm{t}, \mathrm{J}_{\mathrm{HH}}=7.8 \mathrm{~Hz}, 2 \mathrm{H} ; \mathrm{ArH}\right), 7.40-7.42 \mathrm{ppm}(\mathrm{d}, 7.8 \mathrm{~Hz}, 2 \mathrm{H} ; \mathrm{ArH}) ;{ }^{13} \mathrm{C} \mathrm{NMR}$ $\left(150 \mathrm{MHz}, \mathrm{CD}_{3} \mathrm{OD}, 25^{\circ} \mathrm{C} ; \mathrm{CD}_{3} \mathrm{OD}\right): \delta=63.72,127.45,128.53,129.61,130.02$, $131.64,138.48 \mathrm{ppm}$.

Furan-2-methanol (entry 3, Table 1). ${ }^{1} \mathrm{H} \mathrm{NMR}\left(600 \mathrm{MHz}, \mathrm{CDCl}_{3}, 25^{\circ} \mathrm{C}\right.$; TMS): $\delta$ $=2.47(\mathrm{br}, \mathrm{s}, 1 \mathrm{H}, \mathrm{OH}), 4.57\left(\mathrm{~s}, 2 \mathrm{H}, \mathrm{CH}_{2}\right), 6.27\left(\mathrm{~d}, \mathrm{~J}_{\mathrm{HH}}=3.6 \mathrm{~Hz}, 1 \mathrm{H} ; \mathrm{CH}=\mathrm{CH}\right), 6.33$ $\left(\mathrm{dd}, \mathrm{J}_{\mathrm{HH}}=3.0,1.2 \mathrm{~Hz}, 1 \mathrm{H} ; \mathrm{CH}=\mathrm{CH}\right), 7.39\left(\mathrm{~d}, \mathrm{~J}_{\mathrm{HH}}=1.8 \mathrm{~Hz}, 1 \mathrm{H} ; \mathrm{CH}=\mathrm{CH}\right) ;{ }^{13} \mathrm{C} \mathrm{NMR}$ $\left(150 \mathrm{MHz}, \mathrm{CDCl}_{3}, 25^{\circ} \mathrm{C} ; \mathrm{CDCl}_{3}\right): \delta=57.32,107.72,110.35,142.53,154.07 \mathrm{ppm}$.

Citronellol (entry 4, Table 1). ${ }^{1} \mathrm{H} \mathrm{NMR}\left(600 \mathrm{MHz}, \mathrm{CD}_{3} \mathrm{OD}, 25^{\circ} \mathrm{C}\right.$; TMS): $\delta=0.93$ $\left(\mathrm{d}, \mathrm{J}_{\mathrm{HH}}=6.6 \mathrm{~Hz}, 3 \mathrm{H} ; \mathrm{CH}_{3}\right), 1.15-1.22,1.29-1.32\left(\mathrm{~m}, 2 \mathrm{H} ; \mathrm{CH}_{2}\right), 1.35-1.40(\mathrm{~m}, 2 \mathrm{H}$; $\left.\mathrm{CH}_{2}\right), 1.58-1.61(\mathrm{~m}, 1 \mathrm{H} ; \mathrm{CH}), 1.63\left(\mathrm{~s}, 3 \mathrm{H} ; \mathrm{CH}_{3}\right), 1.70\left(\mathrm{~s}, 3 \mathrm{H} ; \mathrm{CH}_{3}\right), 1.98-2.07(\mathrm{~m}, 2 \mathrm{H}$; $\left.\mathrm{CH}_{2}\right), 3.56-3.64\left(\mathrm{~m}, 2 \mathrm{H} ; \mathrm{CH}_{2}\right), 5.13 \mathrm{ppm}\left(\mathrm{t}, \mathrm{J}_{\mathrm{HH}}=7.2 \mathrm{~Hz}, 1 \mathrm{H} ; \mathrm{CH}\right) ;{ }^{13} \mathrm{C}$ NMR $(150$ $\left.\mathrm{MHz}, \mathrm{CD}_{3} \mathrm{OD}, 25^{\circ} \mathrm{C} ; \mathrm{CD}_{3} \mathrm{OD}\right): \delta=17.84,20.05,26.02,26.55,30.41,38.51,40.85$, 61.16, 125.94, $131.84 \mathrm{ppm}$.

Nerol (entry 5, Table 1). ${ }^{1} \mathrm{H}$ NMR $\left(600 \mathrm{MHz}, \mathrm{CD}_{3} \mathrm{OD}, 25^{\circ} \mathrm{C}\right.$; TMS): $\delta=1.63(\mathrm{~s}$, $\left.3 \mathrm{H} ; \mathrm{CH}_{3}\right), 1.70\left(\mathrm{~s}, 3 \mathrm{H} ; \mathrm{CH}_{3}\right), 1.76\left(\mathrm{~s}, 3 \mathrm{H} ; \mathrm{CH}_{3}\right), 2.11\left(\mathrm{~s}, 4 \mathrm{H} ; \mathrm{CH}_{2} \mathrm{CH}_{2}\right), 4.07\left(\mathrm{~d}, \mathrm{~J}_{\mathrm{HH}}=\right.$ $\left.7.2 \mathrm{~Hz}, 2 \mathrm{H} ; \mathrm{CH}_{2}\right), 5.14(\mathrm{~s}, 1 \mathrm{H} ; \mathrm{CH}), 5.38 \mathrm{ppm}\left(\mathrm{t}, \mathrm{J}_{\mathrm{HH}}=6.0 \mathrm{~Hz}, 1 \mathrm{H} ; \mathrm{CH}\right) ;{ }^{13} \mathrm{C} \mathrm{NMR}$ $\left(150 \mathrm{MHz}, \mathrm{CD}_{3} \mathrm{OD}, 25^{\circ} \mathrm{C} ; \mathrm{CD}_{3} \mathrm{OD}\right): \delta=17.77,23.76,25.84,27.82,33.01,59.27$, 
$125.08,125.81,132.77,138.58 \mathrm{ppm}$.

p-tolylmethanol (entry 6, Table 1). ${ }^{1} \mathrm{H}$ NMR $\left(600 \mathrm{MHz}, \mathrm{CD}_{3} \mathrm{OD}, 25^{\circ} \mathrm{C}\right.$; TMS): $\delta$ $=2.33\left(\mathrm{~s}, 3 \mathrm{H} ; \mathrm{CH}_{3}\right), 4.56\left(\mathrm{~s}, 2 \mathrm{H} ; \mathrm{CH}_{2}\right), 7.15\left(\mathrm{~d}, \mathrm{~J}_{\mathrm{HH}}=7.8 \mathrm{~Hz}, 2 \mathrm{H} ; \mathrm{ArH}\right), 7.24 \mathrm{ppm}(\mathrm{d}$, $\left.\mathrm{J}_{\mathrm{HH}}=8.4 \mathrm{~Hz}, 2 \mathrm{H} ; \mathrm{ArH}\right) ;{ }^{13} \mathrm{C} \mathrm{NMR}\left(150 \mathrm{MHz}, \mathrm{CD}_{3} \mathrm{OD}, 25^{\circ} \mathrm{C} ; \mathrm{CD}_{3} \mathrm{OD}\right): \delta=21.23$, $65.17,128.18,130.00,138.03,139.65 \mathrm{ppm}$.

4-Methoxylphenylmethanol (entry 7, Table 1). ${ }^{1} \mathrm{H}$ NMR (400 MHz, $\mathrm{CD}_{3} \mathrm{OD}$, $25^{\circ} \mathrm{C}$; TMS): $\delta=3.77\left(\mathrm{~s}, 3 \mathrm{H} ; \mathrm{CH}_{3}\right), 4.54\left(\mathrm{~s}, 2 \mathrm{H} ; \mathrm{CH}_{2}\right), 6.89\left(\mathrm{~d}, \mathrm{~J}_{\mathrm{HH}}=8.4 \mathrm{~Hz}, 2 \mathrm{H} ; \mathrm{ArH}\right)$, $7.27 \mathrm{ppm}\left(\mathrm{d}, \mathrm{J}_{\mathrm{HH}}=8.4 \mathrm{~Hz}, 2 \mathrm{H} ; \mathrm{ArH}\right) ;{ }^{13} \mathrm{C} \mathrm{NMR}\left(100 \mathrm{MHz}, \mathrm{CD}_{3} \mathrm{OD}, 25^{\circ} \mathrm{C} ; \mathrm{CD}_{3} \mathrm{OD}\right): \delta$ $=54.40,63.59,113.42,128.30,133.32,159.10 \mathrm{ppm}$.

4-Chlorophenylmethanol (entry 8, Table 1). ${ }^{1} \mathrm{H}$ NMR $\left(400 \mathrm{MHz}, \mathrm{CD}_{3} \mathrm{OD}, 25^{\circ} \mathrm{C}\right.$; TMS): $\delta=4.59\left(\mathrm{~s}, 2 \mathrm{H} ; \mathrm{CH}_{2}\right), 7.33-7.39 \mathrm{ppm}(\mathrm{m}, 4 \mathrm{H} ; \mathrm{ArH}) ;{ }^{13} \mathrm{C}$ NMR $(100 \mathrm{MHz}$, $\left.\mathrm{CD}_{3} \mathrm{OD}, 25^{\circ} \mathrm{C} ; \mathrm{CD}_{3} \mathrm{OD}\right): \delta=63.02,128.03,129.87,132.52,140.16 \mathrm{ppm}$.

4-Nitrophenylmethanol (entry 9, Table 1). ${ }^{1} \mathrm{H} \mathrm{NMR}\left(400 \mathrm{MHz}, \mathrm{CD}_{3} \mathrm{OD}, 25^{\circ} \mathrm{C}\right.$; TMS): $\delta=4.74\left(\mathrm{~s}, 2 \mathrm{H} ; \mathrm{CH}_{2}\right), 7.58\left(\mathrm{~d}, \mathrm{~J}_{\mathrm{HH}}=8.8 \mathrm{~Hz}, 2 \mathrm{H} ; \mathrm{ArH}\right), 8.20 \mathrm{ppm}\left(\mathrm{d}, \mathrm{J}_{\mathrm{HH}}=8.4\right.$ $\mathrm{Hz}, 2 \mathrm{H} ; \mathrm{ArH}) ;{ }^{13} \mathrm{C}$ NMR $\left(100 \mathrm{MHz}, \mathrm{CD}_{3} \mathrm{OD}, 25^{\circ} \mathrm{C} ; \mathrm{CD}_{3} \mathrm{OD}\right): \delta=62.65,123.09$, 126.86, 147.01, $149.45 \mathrm{ppm}$.

Methyl 4-(hydroxymethyl)benzoate (entry 10, Table 1). ${ }^{1} \mathrm{H}$ NMR (400 MHz, $\left.\mathrm{CD}_{3} \mathrm{OD}, 25^{\circ} \mathrm{C} ; \mathrm{TMS}\right): \delta=3.90\left(\mathrm{~s}, 3 \mathrm{H} ; \mathrm{CH}_{3}\right), 4.68\left(\mathrm{~s}, 2 \mathrm{H} ; \mathrm{CH}_{2}\right), 7.46\left(\mathrm{~d}, \mathrm{~J}_{\mathrm{HH}}=8.0 \mathrm{~Hz}\right.$, $2 \mathrm{H} ; \mathrm{ArH}) ; 7.98 \mathrm{ppm}\left(\mathrm{d}, \mathrm{J}_{\mathrm{HH}}=8.0 \mathrm{~Hz}, 2 \mathrm{H} ; \mathrm{ArH}\right) ;{ }^{13} \mathrm{C} \mathrm{NMR}\left(100 \mathrm{MHz}, \mathrm{CD}_{3} \mathrm{OD}, 25^{\circ} \mathrm{C}\right.$; $\left.\mathrm{CD}_{3} \mathrm{OD}\right): \delta=51.31,63.19,126.26,129.24,147.14,167.20 \mathrm{ppm}$.

2-Chlorophenylmethanol (entry 11, Table 1). ${ }^{1} \mathrm{H} \mathrm{NMR}\left(400 \mathrm{MHz}, \mathrm{CD}_{3} \mathrm{OD}, 25^{\circ} \mathrm{C}\right.$; TMS): $\delta=4.71\left(\mathrm{~s}, 2 \mathrm{H} ; \mathrm{CH}_{2}\right), 7.24-7.36(\mathrm{~m}, 3 \mathrm{H} ; \mathrm{ArH}), 7.54 \mathrm{ppm}\left(\mathrm{d}, \mathrm{J}_{\mathrm{HH}}=7.6 \mathrm{~Hz}, 1 \mathrm{H}\right.$; $\mathrm{ArH}) ;{ }^{13} \mathrm{C}$ NMR $\left(100 \mathrm{MHz}, \mathrm{CD}_{3} \mathrm{OD}, 25^{\circ} \mathrm{C} ; \mathrm{CD}_{3} \mathrm{OD}\right): \delta=61.03,126.69,128.07$, $128.22,128.76,132.02,138.61 \mathrm{ppm}$. 
Vanillyl alcohol (entry 12, Table 1). ${ }^{1} \mathrm{H} \mathrm{NMR}\left(400 \mathrm{MHz}, \mathrm{CD}_{3} \mathrm{OD}, 25^{\circ} \mathrm{C}\right.$; TMS): $\delta$ $=3.85\left(\mathrm{~s}, 3 \mathrm{H} ; \mathrm{CH}_{3}\right) ; 4.52\left(\mathrm{~s}, 2 \mathrm{H} ; \mathrm{CH}_{2}\right), 6.79(\mathrm{~s}, 2 \mathrm{H} ; \mathrm{ArH}), 6.95 \mathrm{ppm}(\mathrm{s}, 1 \mathrm{H} ; \mathrm{ArH}) ;{ }^{13} \mathrm{C}$ NMR (100 MHz, $\left.\mathrm{CD}_{3} \mathrm{OD}, 25^{\circ} \mathrm{C} ; \mathrm{CD}_{3} \mathrm{OD}\right): \delta=55.04,63.98,110.79,114.66,119.75$, $132.86,145.47,147.54 \mathrm{ppm}$.

3,4,5-Trimethoxyphenylmethanol (entry 13, Table 1). ${ }^{1} \mathrm{H}$ NMR (400 MHz, $\left.\mathrm{CD}_{3} \mathrm{OD}, 25^{\circ} \mathrm{C} ; \mathrm{TMS}\right): \delta=3.75\left(\mathrm{~s}, 3 \mathrm{H} ; \mathrm{CH}_{3}\right), 3.84\left(\mathrm{~s}, 6 \mathrm{H} ; \mathrm{CH}_{3}\right), 4.56\left(\mathrm{~s}, 2 \mathrm{H} ; \mathrm{CH}_{2}\right)$, $6.67 \mathrm{ppm}(\mathrm{s}, 2 \mathrm{H} ; \mathrm{ArH}) ;{ }^{13} \mathrm{C} \mathrm{NMR}\left(100 \mathrm{MHz}, \mathrm{CD}_{3} \mathrm{OD}, 25^{\circ} \mathrm{C} ; \mathrm{CD}_{3} \mathrm{OD}\right): \delta=55.19$, $59.79,63.88,103.65,136.64,137.59,153.06 \mathrm{ppm}$.

2,6-Di-tert-butyl-4-(hydroxymethyl)phenol (entry 14, Table 1). ${ }^{1} \mathrm{H}$ NMR (400 $\left.\mathrm{MHz}, \mathrm{CD}_{3} \mathrm{OD}, 25^{\circ} \mathrm{C} ; \mathrm{TMS}\right): \delta=1.87(\mathrm{~s}, 1 \mathrm{H} ; \mathrm{O}-\mathrm{H}), 1.44\left(\mathrm{~s}, 18 \mathrm{H} ; \mathrm{CH}_{3}\right), 3.73(\mathrm{~s}$, 1H;O-H), 4.50 (s, 2H; $\mathrm{CH}_{2}$ ), 7.17 ppm (s, 2H; ArH); ${ }^{13} \mathrm{C} \mathrm{NMR} \mathrm{(100} \mathrm{MHz,} \mathrm{CD} \mathrm{CD}_{3} \mathrm{O}$, $\left.25^{\circ} \mathrm{C} ; \mathrm{CD}_{3} \mathrm{OD}\right): \delta=29.46,34.13,64.62,123.82,131.83,137.66,153.29 \mathrm{ppm}$.

Cyclohexanol (entry 15, Table 1). ${ }^{1} \mathrm{H} \mathrm{NMR}\left(600 \mathrm{MHz}, \mathrm{CDCl}_{3}, 25^{\circ} \mathrm{C}\right.$; TMS): $\delta=$ $1.19-1.30,1.53-1.56,1.73-1.90\left(\mathrm{~m}, 10 \mathrm{H} ; \mathrm{CH}_{2}\right), 3.60-3.62 \mathrm{ppm}(\mathrm{m}, 1 \mathrm{H} ; \mathrm{CH}) ;{ }^{13} \mathrm{C}$ NMR $\left(150 \mathrm{MHz}, \mathrm{CDCl}_{3}, 25^{\circ} \mathrm{C} ; \mathrm{CD}_{3} \mathrm{Cl}_{3}\right): \delta=24.14,25.46,35.52,70.27 \mathrm{ppm}$.

Cyclopentanol (entry 16, Table 1). ${ }^{1} \mathrm{H}$ NMR (600 MHz, $\mathrm{CDCl}_{3}, 25^{\circ} \mathrm{C}$; TMS): $\delta=$ 1.58 (s, 4H; $\left.\mathrm{CH}_{2} \mathrm{CH}_{2}\right), 1.77 \mathrm{ppm}\left(\mathrm{s}, 4 \mathrm{H} ; \mathrm{CH}_{2}, \mathrm{CH}_{2}\right) ;{ }^{13} \mathrm{C} \mathrm{NMR}\left(150 \mathrm{MHz}, \mathrm{CDCl}_{3}, 25^{\circ} \mathrm{C}\right.$; $\left.\mathrm{CD}_{3} \mathrm{Cl}_{3}\right): \delta=24.22,36.00,74.47 \mathrm{ppm}$

3,5,5-Trimethyl-2-cyclohexen-1-ol (entry 17, Table 1). ${ }^{1} \mathrm{H}$ NMR (600MHz, $\mathrm{CD}_{3} \mathrm{OD}, 25^{\circ} \mathrm{C}$; TMS): $\delta=0.79\left(\mathrm{~s}, 3 \mathrm{H} ; \mathrm{CH}_{3}\right), 0.89\left(\mathrm{~s}, 3 \mathrm{H} ; \mathrm{CH}_{3}\right), 1.12\left(\mathrm{dd}, \mathrm{J}_{\mathrm{HH}}=9.6\right.$, 12Hz, $1 \mathrm{H} ; \mathrm{CH}=\mathrm{CH}), 1.57\left(\mathrm{~s}, 3 \mathrm{H} ; \mathrm{CH}_{3}\right), 1.45-1.52,1.74-1.90\left(\mathrm{~m}, 4 \mathrm{H} ; \mathrm{CH}_{2}\right), 3.96-4.07$ $\left(\mathrm{m}, 1 \mathrm{H} ; \mathrm{CH}_{3}\right), 5.29 \mathrm{ppm}(\mathrm{s}, 1 \mathrm{H} ; \mathrm{CH}=\mathrm{CH}) ;{ }^{13} \mathrm{C} \mathrm{NMR}\left(150 \mathrm{MHz}, \mathrm{CDCl}_{3}, 25^{\circ} \mathrm{C}\right.$; $\left.\mathrm{CD}_{3} \mathrm{Cl}_{3}\right): \delta=23.53,26.24,31.21,44.14,45.29,66.87,123.65,136.05 \mathrm{ppm}$.

1-Phenylethanol (entry 18, Table 1). ${ }^{1} \mathrm{H}$ NMR (600 MHz, $\mathrm{CD}_{3} \mathrm{OD}, 25^{\circ} \mathrm{C}$; TMS): $\delta$ 
$=1.45\left(\mathrm{~d}, \mathrm{~J}_{\mathrm{HH}}=6.6 \mathrm{~Hz}, 3 \mathrm{H} ; \mathrm{CH}_{3}\right), 4,84\left(\mathrm{q}, \mathrm{J}_{\mathrm{HH}}=6.6 \mathrm{~Hz}, 1 \mathrm{H} ; \mathrm{CH}\right), 7.23-7.39(\mathrm{~m}, 5 \mathrm{H}$;

$\mathrm{ArH}) ;{ }^{13} \mathrm{C}$ NMR $\left(150 \mathrm{MHz}, \mathrm{CD}_{3} \mathrm{OD}, 25^{\circ} \mathrm{C} ; \mathrm{CD}_{3} \mathrm{OD}\right): \delta=25.70,70.92,126.53$, 128.14, 129.33, $147.63 \mathrm{ppm}$.

1-(P-tolyl)ethanol (entry 19, Table 1). ${ }^{1} \mathrm{H} \mathrm{NMR}\left(400 \mathrm{MHz}, \mathrm{CD}_{3} \mathrm{OD}, 25^{\circ} \mathrm{C}\right.$; TMS): $\delta=1.45\left(\mathrm{~d}, \mathrm{~J}_{\mathrm{HH}}=6.4 \mathrm{~Hz}, 3 \mathrm{H} ; \mathrm{CH}_{3}\right), 2.31\left(\mathrm{~s}, 3 \mathrm{H} ; \mathrm{CH}_{3}\right), 4.81\left(\mathrm{q}, \mathrm{J}_{\mathrm{HH}}=6.4 \mathrm{~Hz}, 1 \mathrm{H}\right.$; $\mathrm{CH}), 7.13\left(\mathrm{~d}, \mathrm{~J}_{\mathrm{HH}}=8.0 \mathrm{~Hz}, 2 \mathrm{H} ; \mathrm{ArH}\right), 7.27 \mathrm{ppm}\left(\mathrm{d},{ }^{3} \mathrm{~J}_{\mathrm{HH}}=8.0 \mathrm{~Hz}, 2 \mathrm{H} ; \operatorname{ArH}\right) ;{ }^{13} \mathrm{C}$ NMR (150 MHz, $\left.\mathrm{CD}_{3} \mathrm{OD}, 25^{\circ} \mathrm{C} ; \mathrm{CD}_{3} \mathrm{OD}\right): \delta=20.07,24.46,69.37,125.20,128.62$, 136.32, 143.18 ppm.

1-(4-Methoxyphenyl)ethanol (entry 20, Table 1). ${ }^{1} \mathrm{H}$ NMR $\left(600 \mathrm{MHz}, \mathrm{CD}_{3} \mathrm{OD}\right.$, $\left.25^{\circ} \mathrm{C} ; \mathrm{TMS}\right): \delta=1.43\left(\mathrm{~d}, \mathrm{~J}_{\mathrm{HH}}=7.8 \mathrm{~Hz}, 3 \mathrm{H} ; \mathrm{CH}_{3}\right), 3.79\left(\mathrm{~s}, 3 \mathrm{H} ; \mathrm{CH}_{3}\right), 4.79\left(\mathrm{q}, \mathrm{J}_{\mathrm{HH}}=\right.$ $6.6 \mathrm{~Hz}, 1 \mathrm{H} ; \mathrm{CH}), 6.89\left(\mathrm{~d}, \mathrm{~J}_{\mathrm{HH}}=9.0 \mathrm{~Hz}, 2 \mathrm{H} ; \mathrm{ArH}\right), 7.29 \mathrm{ppm}\left(\mathrm{d}, \mathrm{J}_{\mathrm{HH}}=8.4 \mathrm{~Hz}, 2 \mathrm{H}\right.$; $\operatorname{ArH}) ;{ }^{13} \mathrm{C}$ NMR $\left(150 \mathrm{MHz}, \mathrm{CD}_{3} \mathrm{OD}, 25^{\circ} \mathrm{C} ; \mathrm{CD}_{3} \mathrm{OD}\right): \delta=25.51,55.74,70.50,114.69$, $127.77,139.59,160.34 \mathrm{ppm}$.

1-(4-Bromophenyl)ethanol (entry 21, Table 1). ${ }^{1} \mathrm{H}$ NMR (400 MHz, $\mathrm{CD}_{3} \mathrm{OD}$, $\left.25^{\circ} \mathrm{C} ; \mathrm{TMS}\right): \delta=1.42\left(\mathrm{~d}, \mathrm{~J}_{\mathrm{HH}}=6.4 \mathrm{~Hz}, 3 \mathrm{H} ; \mathrm{CH}_{3}\right), 4.81\left(\mathrm{q}, \mathrm{J}_{\mathrm{HH}}=6.4 \mathrm{~Hz}, 1 \mathrm{H} ; \mathrm{CH}\right)$, $5.50(\mathrm{~s}, 1 \mathrm{H} ; \mathrm{OH}), 7.30\left(\mathrm{~d}, \mathrm{~J}_{\mathrm{HH}}=8.4 \mathrm{~Hz}, 2 \mathrm{H} ; \mathrm{ArH}\right), 7.47 \mathrm{ppm}\left(\mathrm{d}, \mathrm{J}_{\mathrm{HH}}=8.4 \mathrm{~Hz}, 2 \mathrm{H}\right.$; $\mathrm{ArH}) ;{ }^{13} \mathrm{C}$ NMR $\left(100 \mathrm{MHz}, \mathrm{CD}_{3} \mathrm{OD}, 25^{\circ} \mathrm{C} ; \mathrm{CD}_{3} \mathrm{OD}\right): \delta=24.18,68.74,120.22$, 127.10, 130.96, $145.58 \mathrm{ppm}$.

Diphenylmethanol (entry 22, Table 1). ${ }^{1} \mathrm{H}$ NMR (600 MHz, $\mathrm{CD}_{3} \mathrm{OD}, 25^{\circ} \mathrm{C}$; TMS): $\delta=5.79(\mathrm{~s}, 1 \mathrm{H} ; \mathrm{H}), 7.22-7.39(\mathrm{~m}, 10 \mathrm{H}) ;{ }^{13} \mathrm{C} \mathrm{NMR}\left(150 \mathrm{MHz}, \mathrm{CD}_{3} \mathrm{OD}, 25^{\circ} \mathrm{C}\right.$; $\left.\mathrm{CD}_{3} \mathrm{OD}\right): \delta=77.01,127.78,128.25,129.31,145.98 \mathrm{ppm}$. 